\title{
Spatial accessibility to basic public health services in South Sudan
}

\author{
Peter M. Macharia, ${ }^{1}$ Paul O. Ouma, ${ }^{1}$ Ezekiel G. Gogo, ${ }^{1}$ Robert W. Snow, ${ }^{1,2}$ \\ Abdisalan M. Noor ${ }^{1,2}$ \\ ${ }^{1}$ Kenya Medical Research Institute/Wellcome Trust Research Programme, Nairobi, Kenya; ${ }^{2}$ \\ Centre for Tropical Medicine and Global Health, Nuffield Department of Clinical Medicine, University \\ of Oxford, Oxford, UK
}

\begin{abstract}
At independence in 2011, South Sudan's health sector was almost non-existent. The first national health strategic plan aimed to achieve an integrated health facility network that would mean that $70 \%$ of the population were within $5 \mathrm{~km}$ of a health service provider. Publically available data on functioning and closed health facilities, population distribution, road networks, land use and elevation were used to compute the fraction of the population within 1 hour walking distance of the nearest public health facility offering curative services. This metric was summarised for each of the 78 counties in South Sudan and compared with simpler metrics of the proportion of the population within $5 \mathrm{~km}$ of a health facility. In
\end{abstract}

Correspondence: Peter M. Macharia, Kenya Medical Research Institute/Wellcome Trust Research Programme, 00100 Nairobi, Kenya. Tel: +254.711 .170493 .

E-mail: pmacharia@kemri-wellcome.org

Key words: South Sudan; Health facilities; Spatial accessibility.

Contributions: PMM, AMN and RWS conceived and designed the experiments; EGG geocoded the health facilities; PMM undertook the analysis with support from POO; PMM, AMN and RWS wrote the paper. All authors agreed with the final manuscript.

Funding: PMM and POO were supported by funding from Wellcome Trust to Kenya the Major Overseas Program (\#107769). Funding from the UK Department for International Development to the INFORM project supports PMM, POO and EGG (\# ITDCZE47). RWS is supported by the Wellcome Trust as Principal Research Fellow (\# 103602). AMN is supported by the Wellcome Trust as an Intermediate Fellow (\#095127). The funders had no role in study design, data collection and analysis, decision to publish, or preparation of the manuscript.

Conflict of interest: the authors declare no potential conflict of interest.

Received for publication: 20 September 2016.

Revision received: 8 March 2017.

Accepted for publication: 8 March 2017.

CCopyright P.M. Macharia et al., 2017

Licensee PAGEPress, Italy

Geospatial Health 2017; 12:510

doi:10.4081/gh.2017.510

This article is distributed under the terms of the Creative Commons Attribution Noncommercial License (CC BY-NC 4.0) which permits any noncommercial use, distribution, and reproduction in any medium, provided the original author(s) and source are credited.
2016, it is estimated that there were 1747 public health facilities, out of which 294 were non-functional in part due to the on-going civil conflict. Access to a service provider was poor with only $25.7 \%$ of the population living within one-hour walking time to a facility and $28.6 \%$ of the population within $5 \mathrm{~km}$. These metrics, when applied sub-nationally, identified the same high priority, most vulnerable counties. Simple metrics based upon population distribution and location of facilities might be as valuable as more complex models of health access, where attribute data on travel routes are imperfect or incomplete and sparse. Disparities exist in South Sudan among counties and those with the poorest health access should be targeted for priority expansion of clinical services.

\section{Introduction}

Access to health services is important for a population's health status and the measurement of its variability is critical to effective allocation of national health resources. Accessibility can be measured on the basis of financial costs to clients, availability of the required resources at health facilities, quality of health care, acceptability and geographic accessibility (Penchansky and Thomas, 1981; Guagliardo, 2004). The latter is the most commonly measured as it is seen as an easily quantifiable and interpretable measure for policy decisions at national and global scales. Spatial accessibility can be defined using indices such as the health facility-to-population ratio or the estimated proportion of population within a specified distance or travel time to health facilities. Clearly, any analysis of spatial accessibility depends critically upon the availability of spatially defined data on the location of health facilities and population (Guagliardo, 2004).

According to the World Health Organization (WHO), child and maternal mortality remain among the highest in the world in South Sudan (WHO, 2014; Valadez et al., 2015). Coincidentally, access to health care is considered one of the poorest in Africa (WHO and World Bank, 2015). The war for independence was one of the longest in the continent (Cometto et al., 2010). At independence in July 2011, public services, especially in the health sector, were virtually non-existent. In an attempt to rapidly improve the situation, the Health Sector Development Programme (HSDP) for 2012-2016 was launched with the ambitious target of ensuring $70 \%$ of the population to have access to health care by 2015 , (Government of South Sudan Ministry of Health, 2012). Civil war re-erupted within South Sudan in December 2013.

In 2012, it was estimated that effective coverage of health services was poor. The World Bank, GSS-MoH and others have estimated that between $40-45 \%$ of the population are settled within 5 $\mathrm{km}$ of a health service provider (Government of South Sudan Ministry of Health, 2012; Downie, 2012; Shabalina, 2014; World 
Bank, 2014). Other reports suggest that between $25-33 \%$ of the population have access to basic and adequate health services (Vogt et al., 2011;WHO, 2009; Cometto et al., 2010). These aggregated national estimates are hard to compare as each has a slightly different meaning and few are accompanied with what data sources used to compute the metrics. Importantly none of these national summaries allow for a more effective understanding of sub-national health access, necessary for planning of effective, equitable resource alocation.

In this study, publically available data on health services, population and transport networks are used within a geographic information system (GIS) to develop spatial models that provide a variety of updated spatial health access metrics nationally and subnationally for South Sudan.

\section{Materials and Methods}

\section{Country and health service context}

South Sudan has an approximate area of $640,000 \mathrm{~km}^{2}$ and is divided into 10 states and 79 counties (including the disputed territory of Abyei), and they represent the first and second levels of administration, respectively (Figure 1) (Government of South Sudan Ministry of Health, 2011, 2012) and covered an estimated population of 12 million people in 2015 according to Southern Sudan Centre for Census Statitistics and Evaluation (SSCCSE) (Southern Sudan Centre for Census Statitistics and Evaluation, 2010; Government of South Sudan Ministry of Health, 2012).

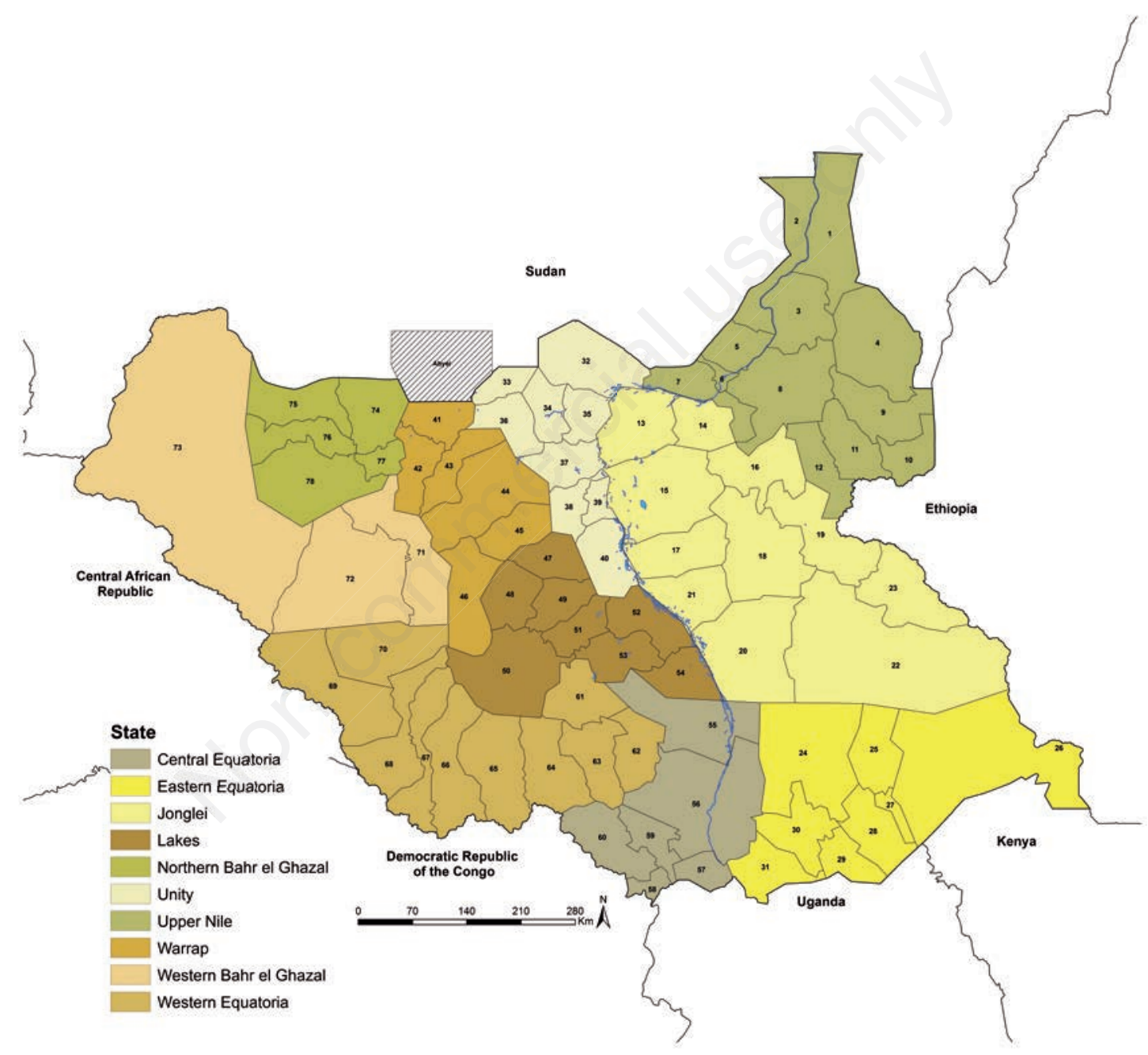

Figure 1. Map of South Sudan counties (n=78) excluding Abyei disputed area across 10 States. County codes are as follows: Upper Nile: Renk (1), Manyo (2), Melut (3), Maban (4), Fashoda (5), Malakal (6), Panyikang (7), Baliet (8), Longochuk (9), Maiwut (10), Luakpiny/Nasir (11), Ulang (12); Jonglei: Fangak (13), Canal/Pigi (14), Ayod (15), Nyirol (16), Duk (17), Uror (18), Akobo (19), Bor South (20), Twic East (21), Pibor (22), Pochalla (23); Eastern Equatoria: Lafon (24), Kapoeta North (25), Kapoeta East (26), Kapoeta South (27), Budi (28), Ikotos (29), Torit (30), Magwi (31); Unity: Pariang (32), Abiemnhom (33), Rubkona (34), Guit (35), Mayom (36), Koch (37), Mayendit (38), Leer (39), Panyijiar (40); Warrap: Twic (41), Gogrial West (42), Gogrial East (43), Tonj North (44), Tonj East (45), Tonj South (46); Lakes: Rumbek North (47), Cueibet (48), Rumbek Centre (49), Wulu (50), Rumbek East (51), Yirol East (52), Yirol West (53), Awerial (54); Central Equatoria: Terekeka (55), Juba (56), Kajo-keji (57), Morobo (58), Lainya (59), Yei (60); Western Equatoria: Mvolo (61), Mundri East (62), Mundri West (63), Maridi (64), Ibba (65), Yambio (66), Nzara (67), Ezo (68), Tambura (69), Nagero (70); Western Bahr el Ghazal: Jur River (71), Wau (72), Raga (73); and Northern Bahr el Ghazal: Aweil East (74), Aweil North (75), Aweil West (76), Aweil South (77) and Aweil Centre (78). 
Approximately $90 \%$ of the population live in rural areas (Southern Sudan Centre for Census Statitistics and Evaluation, 2010; Government of South Sudan Ministry of Health, 2012).

The formal health services in South Sudan are provided mainly by the Government supported by non-governmental organizations (NGOs) and faith based organizations (FBOs) (Government of South Sudan Ministry of Health, 2013). The public health sector is structured along four tiers: primary health care units (PHCUs), primary health care centres (PHCCs), county hospitals (CHs), state hospitals (SHs) and teaching hospitals (THs) (Government of South Sudan Ministry of Health, 2012). PHCUs are the first level of primary care and provide basic preventive, promotive and curative services and expected to serve a population of 15,000. PHCCs, aimed at serving a population of 50,000, are the immediate reference facilities for the PHCUs, providing all the services provided by a PHCU but in theory additional services covering diagnostic laboratory, maternity and inpatient care. The CHs are referral facilities for PHCCs and provide emergency services aimed at serving a maximum population of 300,000 people, while SHs are designed to serve a population of 500,000 people. CHs and SHs represent the secondary health care level while THs provide tertiary care. Although, according to the national policy health care is decentralised with governance at the national, state and county levels (Government of South Sudan Ministry of Health, 2011), the national Government still retains most regulatory, budgetary and commodity supply responsibilities. The private sector is in a nascent stage with a few private clinics, mostly in urban areas, but they are poorly equipped (Cornelis and Ron, 2006).

\section{Spatial databases}

\section{Health facilities}

A health facility database was obtained from the Humanitarian Data Exchange Portal (HDX) of the United Nations Office for the Coordination of Humanitarian Affairs (UN-OCHA) (UN-OCHA, 2016a) developed originally from a mapping exercise undertaken by the $\mathrm{MoH}$ and the World Bank in 2009 and regularly updated by UN-OCHA. The original database had 1877 health facilities, with the majority of these facilities (78.5\%) already mapped using global positioning system (GPS) receivers. For those that had not been spatially positioned, coordinates were derived through geocoding of place or village names, using a settlement dataset of South Sudan (UN OCHA, 2016b) and online sources including Google Earth (http://www.google.co.uk/intl/en_uk/earth/) and Geo-names (http://www.geonames.org/search.html?q=\&country=SS=). Duplicate facilities were removed, those labelled non-operational due to conflict, destruction and lack of resources, private, or reserved for specialised care or security forces were also removed. The final database covered all NGO, FBO and Government services capable of offering the basic preventive, promoter and curative health services to the general public.

\section{Population}

Critical to the modelling of spatial accessibility to services is a reliable understanding of the distribution and density of the populations they serve. The last census in South Sudan was undertaken in 2008 and data are not available below county levels. To overcome this low-resolution population data, WorldPop (http://www.worldpop.org.uk/) has developed spatial disaggregation techniques to redistribute population counts to finer spatial resolutions (Linard et al., 2012; Stevens et al., 2015). These mod- els for South Sudan have reallocated populations within census units by disaggregating population count data from the 79 counties in 2008 to a $100 \mathrm{~m}$ spatial resolution grid using Land cover class population densities derived from $30 \mathrm{~m}$ Landsat satellite imagery (http://landsat.visibleearth.nasa.gov/) as weights using dasymetric modelling techniques (Mennis, 2009; Linard et al., 2011). Each grid was then projected to match the UN national population estimates for the year 2015 (United Nations Population Division, 2015). Population distribution, according to the WorldPop predictions highlights the over-dispersion of settlements in South Sudan, with the highest concentration of people in southern most counties in Central Equatoria State, and in the states of Warrap and Northern Bahr el Ghazal (Figure 2A).

\section{Road network}

People mostly travel on road networks to reach services rather than along straight lines (Euclidean) from their homes to the point of service provision. Therefore, a road surface was developed as a composite of three online sources: Global Roads Open Access Data Set, assembled mostly from Vector Smart Map Level 0 (VMap0), Edition 5 for the period 1980 - 2010 (Center for International Earth Science Information Network, 2013); the HDX portal, which sourced data from the World Food Programme and last updated in December 2012 (UN OCHA, 2016c); and from Open Street Map which is mapped and maintained by volunteers all over the world (OpenStreetMap, 2015). The three road network data were merged and duplicates removed in ArcGIS, version 10.1 (ESRI Inc., Redlands, CA, USA). The resultant database of the road network was exported to Google Earth and all visually identifiable roads and footpaths digitised in areas where they had not been captured. The road network across the country is patchy, of $17,000 \mathrm{~km}$, only $200 \mathrm{~km}$ is paved road (World Bank, 2016). During the dry season most of unpaved roads are only accessible by allterrain vehicles, while during the rainy season most roads are impassable (UN OCHA, 2014a) (Figure 2B).

\section{Land cover and use}

Satellite-derived information is available on land cover and land use, identifying properties of the geographical space people need to traverse. Data were obtained from the GlobCover (http://maps.elie.ucl.ac.be/CCI/viewer/) for the 2010 epoch (20082012) that used the processed $300-\mathrm{m}$ and $1-\mathrm{km}$ spatial resolution Medium Resolution Spectrometer (MERIS) (https:/earth.esa.int/ web/guest/missions/esa-operational-eo-missions/envisat/instruments/meris) and SPOT-VEGETATION (http:/www.vgt.vito.be/) sensors respectively. The hierarchical classification used is based on United Nations Land Cover Classification System of the Food and Agricultural Organization (FAO) (FAO, 2000). The topography of the country is less varied with shrub land and broadleaved, deciduous, open tree cover dominant across most parts with a stretch of shrub/herbaceous cover, brackish flooded water along the edges of River Nile (Figure 2C).

\section{Digital elevation model}

One impedance to walking travel times is slope (Tobler, 1993). To assemble elevations above sea level the Advanced Space borne Thermal Emission and Reflection Radiometer (ASTER) Global Digital Elevation Model Version 2 (GDEM V2) (https://asterweb. jpl.nasa.gov/gdem.asp) was used at a spatial resolution of $30 \mathrm{~m}$ (Figure 2D). 


\section{Analysis of spatial accessibility}

Two measures of spatial accessibility were computed: the proportion of population by county within $5 \mathrm{~km}$ Euclidean distance to any public health facility and the proportion of population by county within one hour travel time to any public health facility. Sub-national descriptions of populations to facility ratios are less valuable as people seek treatment across county boundaries, so while useful as a measure of national health access, for policy targets and international comparisons, it is less valuable for subnational mapping of vulnerability.

\section{Euclidean distances}

The location of each health facility was then used to develop a $100 \times 100 \mathrm{~m}$ spatial resolution surface of Euclidean distance to the nearest health facility using the Euclidean Distance Tool in ArcGIS. The proportion of population within 5-km Euclidean distance was extracted from the gridded population surface using the Zonal statistics Tool in ArcGIS for each county.

\section{Travel time}

The topologically defined road network was converted into raster surface of roads matching that of the land cover surface. The rasterised road surface and the land cover classes were then assigned travel time equivalent to the number of hours taken to cross each cell based on the type. All the roads were assigned a walking speed of 5 $\mathrm{km} / \mathrm{h}$ (Noor et al., 2006; Ray and Ebener, 2008), while different land cover features were assigned different recommended walking speeds ranging from $1 \mathrm{~km} / \mathrm{h}$ across tree cover and flooded areas to $5 \mathrm{~km} / \mathrm{h}$ across shrub land (Alegana et al., 2012). Slope derived from digital elevation model (DEM) was used to calculate the actual surface distance covered between contiguous cells and to adjust the walking speed by decreasing the up-slope and down-slope speed with increase in slope, while slightly increasing the speed for a slightly negative slope when walking down-slope using the Path Distance Tool of ArcGIS. For travel time analysis, only walking distances to the nearest health facilities were computed, based upon the fact that $90 \%$ of the population are rural and have been reported to have very

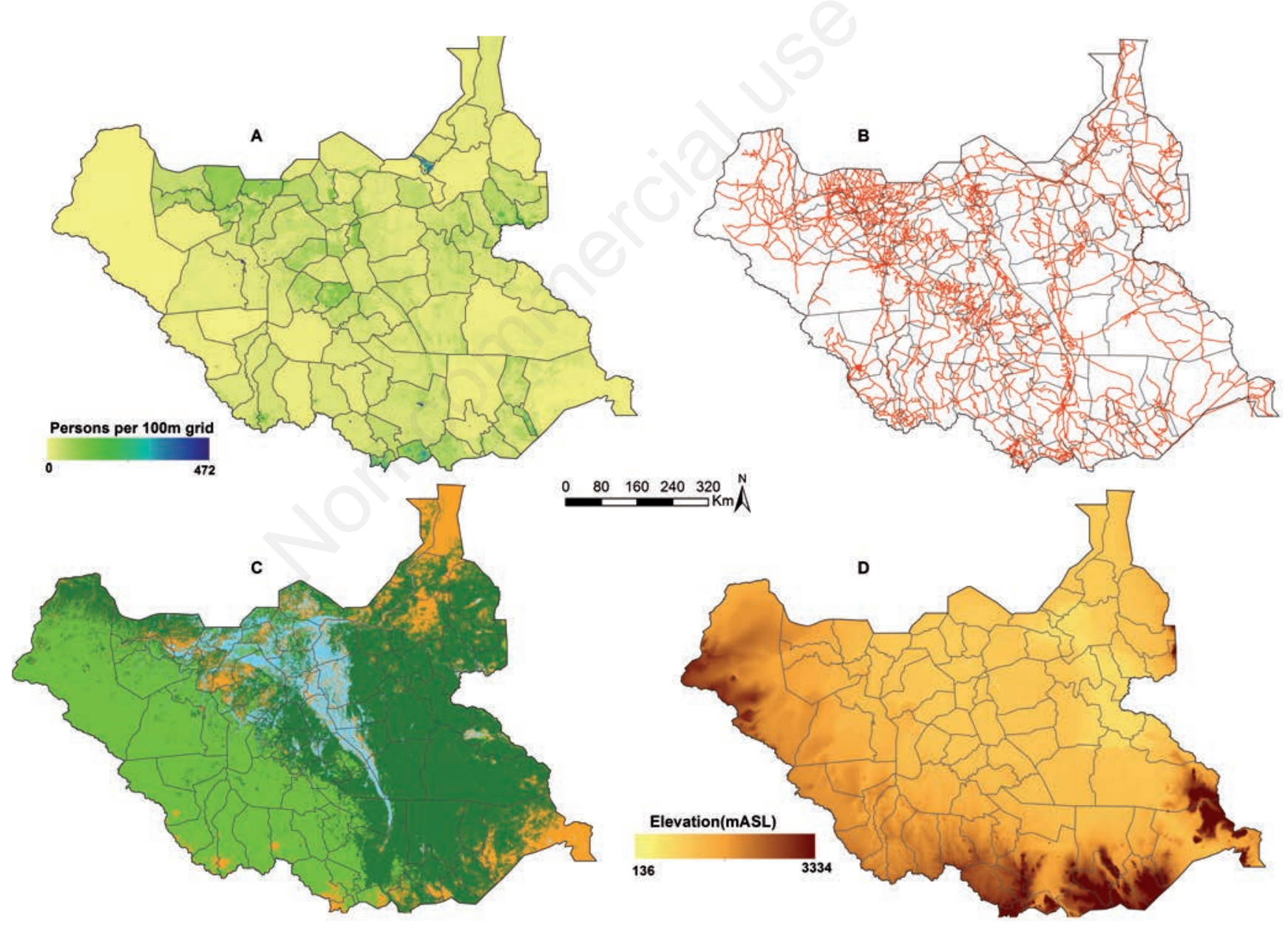

Figure 2. A) Modelled population distribution at $100 \times 100 \mathrm{~m}$ spatial resolution; B) road network; C) major land cover classes: shrub land dark green $(46.5 \%)$, tree cover (open deciduous broadleaved) green $(35.1 \%)$, shrub/herbaceous cover flooded with fresh/saline/brackish water blue $(7.5 \%)$, and others brown $(10.9 \%)$; D) altitude measured by digital elevation model [metres above the mean sea level (m asl)] with an increase in elevation from light yellow (136 m asl) to dark brown (3334 $\mathrm{m}$ asl). 
low access to vehicular transport because of the very poor road infrastructure (Downie, 2012; GSS-MOH, 2012; UN OCHA, 2012). The walking time to the health facilities was estimated from each population grid by identifying the shortest distance to the nearest health facility. The total walking time was then computed by adding up the time needed to cross-adjoining cells to the nearest health facility using the Path Distance Tool of ArcGIS. The proportion of population within one hour was extracted using the Zonal Statistic Tool of ArcGIS at the county level.

\section{Results}

\section{Health service distribution}

After careful comparisons of health facilities' spatial locations and names, duplicates (70), services dedicated for military personnel (27), specialist services (18), private facilities (15) and health facilities that were reported to be non-functional (294) were removed. Those defined as non-functional ranged from $5 \%$ in Western Equatoria State to over 23\% in the states of Jonglei, Lakes and Upper Nile, and nearly $33 \%$ in Unity State (Figure 3). Three hospitals had been affected by the conflict and were defined as non-functional. Out of the 1446 geo-coded public services regarded as functional around 2016, 27, 7 and 3 were county, state and tertiary hospitals, respectively, 286 were PHCCs and 1123 were PHCUs. The distribution of the functioning public sector facilities alongside those that have been closed is shown in Figure 3 with data provided in the Appendix.

Nationally, across all facility types, the facility-to-population ratio was about 1 facility offering curative services for 7947 people. Hospitals served on average 312,000 people, each PHCC 40,373 people and each PHCU 10,218 people.

\section{Euclidean distance}

Only about $28.6 \%$ the population were within $5 \mathrm{~km}$ Euclidean distance to the nearest public health facility. Without much vehicular transport only $7.7 \%$ of the population are within $5 \mathrm{~km}$ of a county, state or national referral hospital. Sub-nationally, the county level aggregated data based on Euclidean distance of population to the nearest facility shows that there are only three Counties (Kajo Keji, Yei and Torit) that have reached the $70 \%$ target set by the $\mathrm{MoH}$ to be achieved by 2015 (Government of South Sudan

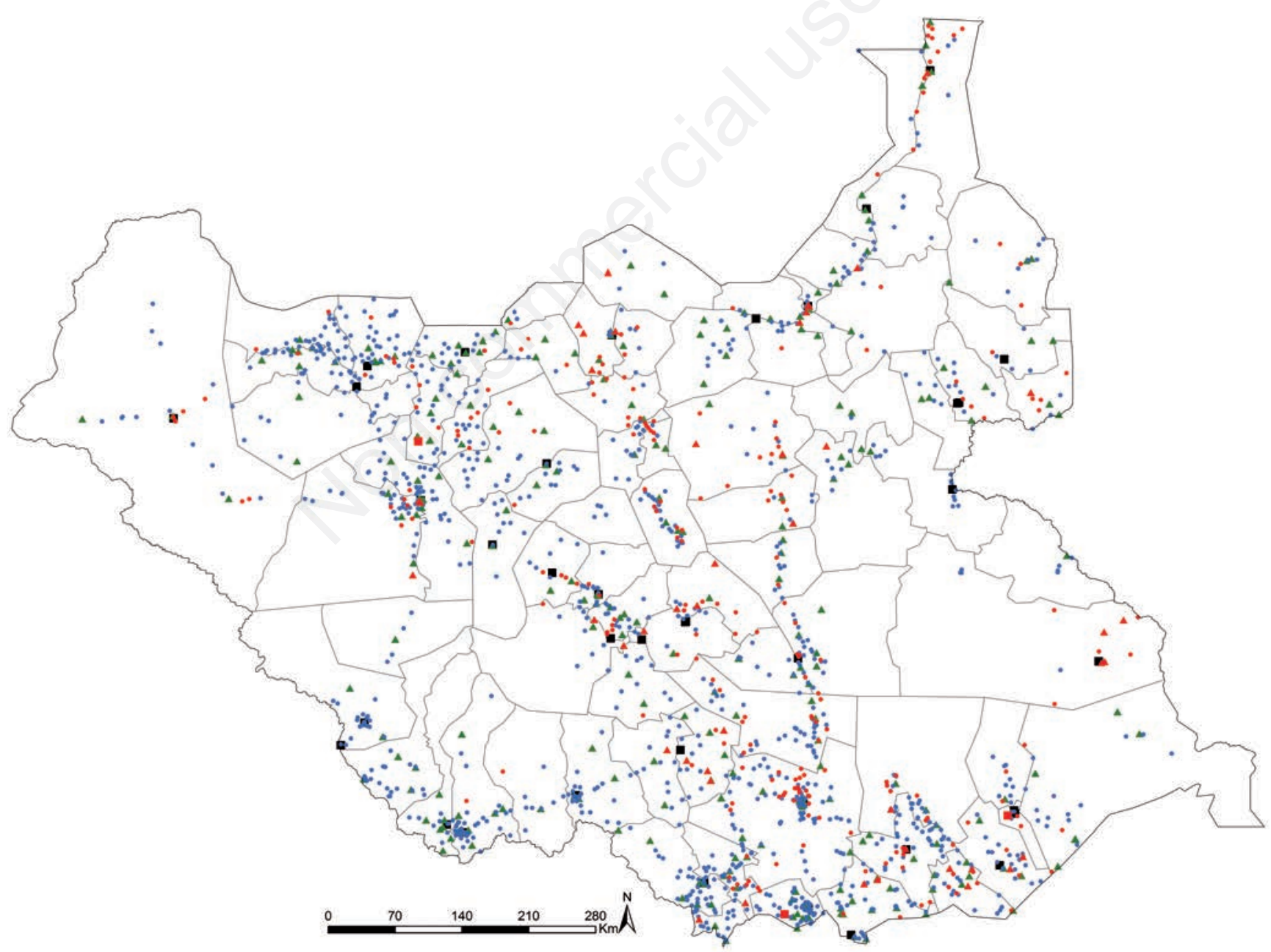

Figure 3. Distribution of functional ( $n=1453)$ and non-functional ( $n=294$; shown in red) health facilities; Primary Health Care Unit (dot), Primary Health Care Centre (triangle) and Hospital (square). 
Ministry of Health, 2012). 71 (91\%) of the 78 counties have less than $50 \%$ of their populations within $5 \mathrm{~km}$ of a functioning health facility (Figures 4A and 5). 21 (27\%) counties, where 10 are located in Upper Nile and Jonglei States, are most vulnerable with less than $10 \%$ of their population within a $5 \mathrm{~km}$ Euclidean radius of a public health facility providing clinical service (Figures 4A and 5).

\section{Travel time}

The proportion of the population at each $1 \times 1 \mathrm{~km}^{2}$ grid was assigned travel times to their nearest facility based upon a composite walking time allowing for roads access, land cover and elevation. Overall, the proportion of the population living with an estimated one hour walking time to the nearest facility was $25.7 \%$.

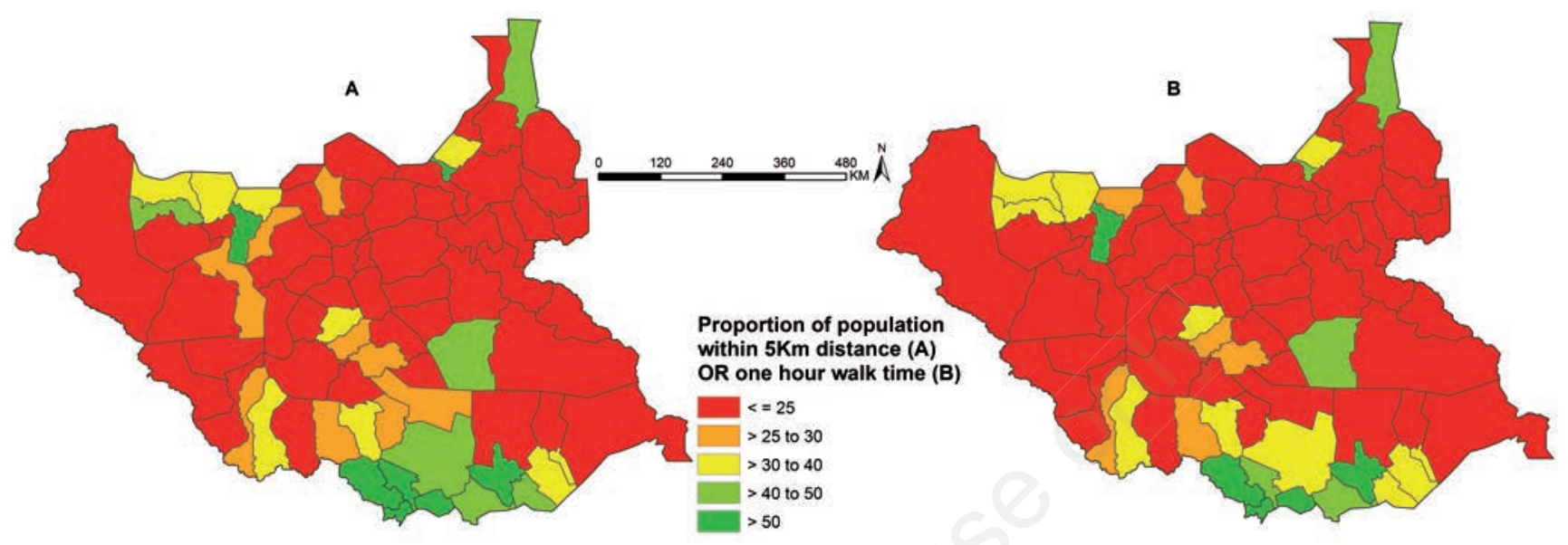

Figure 4. A) Proportion of population within $5 \mathrm{~km}$ radius of the nearest functional public health facility and (B) proportion of population within 1 hour of walking time to the nearest functional public health facility.

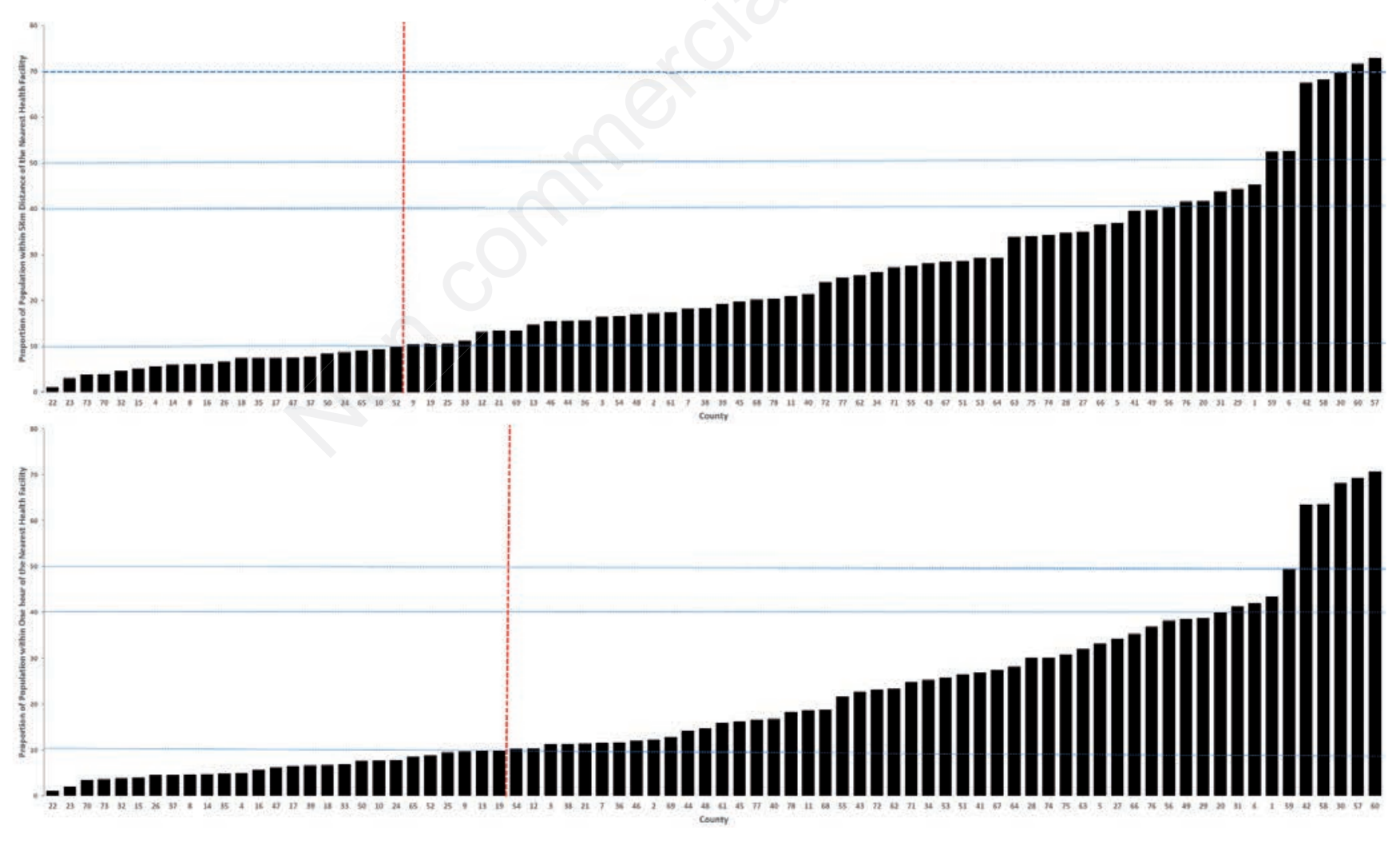

Figure 5. Ranked accessibility indices per county from poorest to least poor with numbers on $X$ axis corresponding to map shown in Figure 1. Top panel is based on proportion of population within a Euclidean distance of $5 \mathrm{~km}$ radius of health facility; bottom panel is the proportion of the population within 1 hour walking time to nearest health facility. Dotted horizontal lines are $70 \%$ (Health Sector Development Programme target on top panel), $50 \%, 40 \%$ (previously described access) and $10 \%$ proportions of population within 5 km Euclidean distance (top panel) and 1-hour walking time (bottom panel). Vertical dotted line represents most vulnerable counties. 
Over $22 \%$ of the population had a walking travel time to the nearest clinical service of more than 5 hours. Sub-nationally, only five counties had more than $50 \%$ of their populations within a 1 hour walking time to the nearest facility, $26(33 \%)$ counties had less than $10 \%$ of their population living within 1 hour of walking to a facility (Figures 4B and 5). The 26 poorest access counties corresponded largely with those described using Euclidean distance methods (Figure 5).

\section{Discussion}

Only $28.6 \%$ of the population are within $5 \mathrm{~km}$ of a public health curative service, a long way from the HSDP's ambition of 70\% (Government of South Sudan Ministry of Health, 2012). More than $74 \%$ of the population live more than 1 hour of walking away from a public health facility. The findings suggest that the situation is much worse than previously reported (WHO, 2009; Cometto et al., 2010; Government of South Sudan Ministry of Health, 2011, 2012; Vogt et al., 2011; Downie, 2012; Shabalina, 2014; World Bank, 2014). Here, high-resolution publically available data have been used to develop widely used metrics of spatial access to health services and used to describe the sub-national disparities in access. While the picture overall is one of poor access, across all metrics presented, it is worse in some specific counties (Figures 4 and 5), notably the counties of Pibor, Pochalla, Nagero, Raga and Paring. Since December 2013, when the current civil war started, there have been major disruptions of public services, affecting an already fragile health system (Berendes et al., 2014; Green, 2014; UN OCHA 2014b; nternational Organization for Migration, 2016) contributing to the closure of many facilities (Figure 3). Despite multilayered modelling of travel times, using more complex levels of analysis, counties with the poorest access were identified equally with simpler measures of Euclidean distance metrics (Figures 4 and 5). Approaches to modelling spatial access have grown over the last decade, inlcuding gravity models such as kernel density (Guagliardo et al., 2004) or two-step floating catchment area methods (Radke and Mu, 2000; Luo and Qi, 2009). However, all these depend intrinsically on the quality of input data, notably the accuracy of health service locations, population, travel routes and an understanding of travel modes. In studies whose aim is to compare relative access to care as opposed to a description of elaborate journey paths, Euclidean distance might be appropriate and can be used sub-nationally to guide effective allocation of resources to meet apparent unmet needs (Nesbitt et al., 2014; Gautam et al., 2014). As shown in the present study (Figures 4 and 5) Euclidean distance was comparable to the more complex travel time models allowing appropriate identification of vulnerable counties. There are obvious caveats to the analysis of the data assembled here. Firstly, there will be a huge uncertainty in the maps of population distribution and density used to compute the geographic coverage of health services. These have used old census input data and do not reflect the post-independence human settlement. Settlement maps have been developed by OCHA to support humanitarian assistance (UN OCHA, 2016b), but these do not have population counts and would fluctuate almost daily within the context of the current civil strife. However, more data are available than currently used by WorldPop to provide a more reliable population surface. Secondly, there is a paucity of detailed information on the travel modes populations frequently use to reach health facilities and these vary in times of conflict and pre- cisely how much non-walking transport is available. It is also probably true that the population minority living in urban areas access services differently to those in rural areas. Finally, the assumption in the travel-time models is that people use the nearest health facility, which ignores other influences on choice of service provider (Gulliford et al., 2002). These combined factors, which cannot be addressed with current data, have a greater influence on the traveltime in the models developed compared to the Euclidean models.

\section{Conclusions}

Spatial accessibility to health services in South Sudan remains very low, with an estimated $71 \%$ of the population living in areas outside $5 \mathrm{~km}$, which is approximately equivalent to 1 hour from a public health facility. This low spatial accessibility is primarily a function of the inadequate distribution of health facilities, but also the inoperability of over 294 health facilities. The analysis presented here suggests that the simpler Euclidean distance models probably perform as well compared to more complex models. The metrics may be used by the South Sudan $\mathrm{MoH}$ to plan the distribution of resources more equitably to improve access to care and by humanitarian agencies to strategically invest in the dysfunctional counties that have suffered from conflict.

\section{References}

Alegana V, Wright JA, Petrina U, Noor AM, Snow RW, Atkinson PM, 2012. Spatial modelling of healthcare utilisation for treatment of fever in Namibia. Int J Health Geogr 11:6.

Berendes S, Lako RL, Whitson D, Gould S, Valadez JJ, 2014. Assessing the quality of care in a new nation: South Sudan's first national health facility assessment. Trop Med Int Health 19:1237-48.

Center for International Earth Science Information Network, 2013. Global Roads Open Access Data Set, Version 1 (gROADSv1). Center for International Earth Science Information Network, New York, NY, USA. Available from: http://sedac.ciesin. columbia.edu/ data/set/groads-global-roads-open-access-v1

Cometto G, Fritsche G, Sondorp E, 2010. Health sector recovery in early post conflict environments: experience from southern Sudan. Disasters 34:885-909.

Cornelis R, Ron W, 2006. Health in fragile states, country case study: Southern Sudan. Basic Support for Institutionalizing Child Survival (BASICS) for the United States Agency for International Development (USAID), Arlington, VA, USA. Available from: pdf.usaid.gov/pdf_docs/Pnadr481.pdf

Downie R, 2012. The state of public health in South Sudan: a critical condition. Center for Strategic and International Studies, Washington, DC, USA.

FAO, 2000. Land Cover Classification System (LCCS): classification concepts and user manual. Food and Agriucultural Organizantion, Rome, Italy. Available from: www.fao.org/ docrep/003/ x0596e/x0596e00.HTM

Gautam S, Li Y, Johnson TG, 2014. Do alternative spatial healthcare access measures tell the same story? GeoJournal 79:223-35.

Government of South Sudan Ministry of Health, 2011. Health Sector Development Plan 2011-2015: transforming the health system for improved services and better coverage. Government 
of South Sudan Ministry of Health, Juba, South Sudan.

Government of South Sudan Ministry of Health, 2012. Health Sector Development Plan 2012-2016: one maternal death is too many. Government of South Sudan Ministry of Health, Juba, South Sudan.

Government of South Sudan Ministry of Health, 2013. The reproductive health policy: present and future prosperity through safe motherhood and healthy childhood. Government of South Sudan Ministry of Health, Juba, South Sudan.

Green A, 2014. Fighting restricts access to health care in South Sudan. Lancet 384:1252.

Guagliardo MF, 2004. Spatial accessibility of primary care: concepts, methods and challenges. Int J Health Geogr 3:3-10.

Guagliardo MF, Ronzio CR, Cheung I, Chacko E, Joseph JG, 2004. Physician accessibility: an urban case study of pediatric providers. Health Place 10:273-83.

Gulliford M, Figueroa-Munoz J, Morgan M, Hughes D, Gibson B, Beech R, Hudson M, 2002. What does "access to health care" mean? J Health Serv Res Policy 7:186-8.

International Organization for Migration, 2016. South Sudan consolidated appeal: January-December 2016. Available from: http://reliefweb.int/sites/reliefweb.int/files/resources/2016IOM-SouthSudan-Consolidated-Appeal.pdf

Linard C, Gilbert M, Snow RW, Noor AM, Tatem AJ, 2012. Population distribution, settlement patterns and accessibility across Africa in 2010. PLoS One 7:e31743.

Linard C, Gilbert M, Tatem AJ, 2011. Assessing the use of global land cover data for guiding large area population distribution modelling. GeoJournal 76:525-38.

Luo W, Qi Y, 2009. An enhanced two-step floating catchment area (E2SFCA) method for measuring spatial accessibility to primary care physicians. Health Place 15:1100-7.

Mennis J, 2009. Dasymetric mapping for estimating population in small areas. Geogr Compass 3:727-45.

Nesbitt RC, Gabrysch S, Laub A, Soremekun S, Manu A, Kirkwood BR, Amenga-Etego S, Wiru K, Höfle B, Grundy C, 2014. Methods to measure potential spatial access to delivery care in low- and middle-income countries: a case study in rural Ghana. Int J Health Geogr 13:25.

Noor AM, Amin AA, Gething PW, Atkinson PM, Hay SI, Snow RW, 2006. Modelling distances travelled to government health services in Kenya. Trop Med Int Heal 11:188-96.

OpenStreetMap, 2015. Planet. Available from: http://extract. bbbike.org/

Penchansky R, Thomas JW, 1981. The concept of access: definition and relationship to consumer satisfaction. Med Care 19:127-40.

Radke J, Mu L, 2000. Spatial decompositions, modeling and mapping service regions to predict access to social programs. Ann GIS 6:105-12.

Ray N, Ebener S, 2008. AccessMod 3.0: computing geographic coverage and accessibility to health care services using anisotropic movement of patients. Int J Health Geogr 7:63.

Shabalina J, 2014. Critical diagnosis: the case for placing South Sudan's healthcare system at the heart of the humanitarian response. Available from: http://insights.careinternational. org.uk/media/k2/ attachments/Critical-Diagnosis-South-Sudanhealthcare-report-Dec-2014.pdf

Southern Sudan Centre for Census Statitistics and Evaluation, 2010. Statistical yearbook for Southern Sudan. Southern Sudan Centre for Census Statitistics and Evaluation, Juba, South
Sudan. Available from: http://static1.1.sqspcdn.com/ static/f/750842/11453911/ 1301368226337/Statistical+Year + Book+For+Southern+Sudan+2010+Final.pdf?token=3zyXiL gVNrW1Iemr2rjK6e6ljsA\%3D

Stevens FR, Gaughan AE, Linard C, Tatem AJ, 2015. Disaggregating census data for population mapping using random forests with remotely-sensed and ancillary data. PLoS One 10:e0107042.

Tobler W, 1993. Three presentations on geographical analysis and modeling: non-isotropic geographic modeling; speculations on the geometry of geography. Global Spatial Analysis, Santa Barbara, CA, USA.

UN OCHA, 2012. South Sudan consolidated appeal 2013. Available from: http://docs.unocha.org/sites/dms/CAP/ CAP 2013_South_Sudan.pdf

UN OCHA, 2014a. South Sudan concept of operations. Available from: http://reliefweb.int/sites/reliefweb.int/files/resources/ 793ae408-95bd-4e79-84ec-29b31 eca4b6d.pdf

UN OCHA, 2014b. South Sudan humanitarian response plan 2015. Available from: reliefweb.int/sites/reliefweb.int/files/ resources /SOUTH\%20SUDAN\%20HRP\%202015_0.pdf

UN OCHA, 2016a. South Sudan: health facilities. Available from: https://data.humdata.org/dataset/south-sudan-health

UN OCHA, 2016b. South Sudan settlements. Available from: https://data.humdata.org/dataset/sudan-settlements

UN OCHA, 2016c. South Sudan: roads. Available from: https://data.humdata.org/dataset/south-sudan-roads

United Nations Population Division, 2015. World population prospects: the 2015 revision, data booklet. United Nations Population Division, New York, NY, USA. Available from: https://esa.un.org/unpd/wpp/Publications/Files/WPP2015 DataBooklet.pdf

Valadez JJ, Berendes S, Lako R, Gould S, Vargas W, Milner S, 2015. Finding the gap: revealing local disparities in coverage of maternal, newborn and child health services in South Sudan using lot quality assurance sampling. Trop Med Int Health 20:1711-21.

Vogt F, Heudtlass P, Guha-sapir D, 2011. Health data in civil conflicts: South Sudan under scrutiny. CRED, Brussels, Belgium. Available from: cred.be/sites/default/files/WP_282.pdf

WHO, 2009. Country Cooperation Strategy for WHO and Sudan 2008-2013. Available from: apps.who.int/iris/bitstream/ 10665/ 113233/1/CCS_Sudan_2010_EN_14477.pdf

WHO, 2014. South Sudan: WHO statistical profile. http://www.who.int/ gho/countries/ssd.pdf?ua=1

WHO and World Bank, 2015. Tracking universal health coverage: first global monitoring report. Available from: http://www.who.int/ healthinfo/universal_health_coverage/ report/2015/en/

World Bank, 2014. A health project in South Sudan is helping provide critical services for women and children in the midst of conflict. Available from: http://www.worldbank. org/en/news/ feature/ 2014/03/06/a-health-project-in-south-sudan-is-helping-provide-critical-services-for-women-and-children-in-themidst-of-conflict

World Bank, 2016. A triumph over long odds: building rural roads in South Sudan. Available from: http://www.worldbank.org/ en/news/feature/2016/02/09/a-triumph-over-long-odds-building-rural-roads-in-south-sudan 